\title{
Low activity sn-glycerol-3-phosphate dehydrogenase variants in natural populations of Drosophila melanogaster
}

\author{
JOHN B. GIBSON, ANH CAO, JANE SYMONDS \& DARRYL REED \\ Molecular and Population Genetics Group, Research School of Biological Sciences, The Australian National University, \\ P.O. Box 475, Canberra City, ACT 2601, Australia
}

\begin{abstract}
Eleven Australian natural populations of Drosophila melanogaster were screened by electrophoresis for evidence of null and/or low activity variants at the Gdph locus. Of 5018 alleles investigated, 57 had markedly lower GPDH activity, as judged by their heterozygous phenotypes, than control alleles. GPDH assays of 13 of these variant alleles showed that whilst they were heterogeneous in their properties and included electrophoretic variants and two categories of low-activity variant, there were no true null alleles. The most common low-activity variants exhibited dominance for GPDH activity in heterozygotes with normal alleles, and were shown to share this property with an allele previously isolated from a London (UK) population (Langley et al., 1981).
\end{abstract}

Keywords: Drosophila melanogaster, low-activity, sn-glycerol-3-phosphate dehydrogenase.

\section{Introduction}

Genetic variation in natural populations is evidence of past mutational events and is the raw material for adaptive evolution. One question concerns the relationships between the amount of this variation, its phenotypic effects and the pattern of evolution of specific proteins. Relevant data can be obtained for gene-enzyme systems in those eukaryotes in which it is possible to integräte population genetic studies with biochemical and systematic information. A pertinent example is the dimeric enzyme $s n$-glycerol-3-phosphate dehydrogenase ( $\alpha$ GPDH, NAD ${ }^{+}$, E.C.1.1.1.8) which has an important role in the Dipteran 'glycerophosphate cycle' for the rapid production of energy required for flight (Sacktor, 1970). Evidence from microcomplement fixation studies suggests that, within the Diptera, GPDH evolves relatively slowly, at a rate similar to that of cytochrome $c$ (Collier \& MacIntyre, 1977a). In addition there is a considerable amino acid sequence similarity between the Drosophila melanogaster enzyme and that from rabbit muscle (Otto et al., 1980; Chambers et al., 1985), which shows that the primary structure of GPDH has been conserved over long periods of evolutionary time.

Within the species $D$. melanogaster, populations are commonly polymorphic for only two electrophoretically distinguishable alleles, $G p d h^{F}$ and $G p d h^{S}$ (Grell,
1967; Johnson \& Schaffer, 1972; Oakeshott et al., 1982; Singh et al., 1982), and surveys which utilize a variety of electrophoretic methods and thermostability studies have not detected extra genetic variation at the $G p d h$ locus (Coyne et al., 1979; Bewley, 1978; LaurieAhlberg \& Bewley, 1983), other than a rare mobility variant, Gpdh ${ }^{U F}$ (Jiang et al., 1989).

In contrast to these data, which suggest little population variation at the Gpdh locus, Voelker et al. (1980a) and Langley et al. (1981) reported that, in two surveys aimed at assessing null allele frequencies at 25 allozyme loci in D. melanogaster populations, the highest frequency of null alleles ( 0.83 per cent) was at the Gpdh locus, when the average frequency over all loci was 0.24 per cent. It was subsequently shown that the nulls at the Gpdh locus were more heterogeneous in their properties than the nulls at any other locus (Burkhart et al., 1984). A recent survey by Freeth \& Gibson (1985) observed even higher frequencies of putative Gpdh null alleles, ranging from 0.2 to 2.7 per cent, with an average of 1.6 per cent, in six natural populations of $D$. melanogaster sampled in Tasmania, Australia. In the same surveys, alcohol dehydrogenase $(A d h)$ null alleles were found at an average frequency of 1.3 per cent. These values, which were markedly higher at both loci than those found by Langley et al. (1981), prompted questions about the origins and kinds of null alleles at the two loci in natural popula- 
tions. Analyses of a number of $A d h$ null alleles extracted from the Tasmanian populations showed that they all lacked ADH CRM (Freeth et al., 1986; 1987), and probably shared a common origin (Jiang et al., 1988; Gibson \& Wilks, 1989).

To test whether the Tasmanian frequencies were atypical, surveys of $G p d h$ variant allele frequencies were made in 11 Australian populations of $D$. melanogaster. This paper describes these data, which show that $G p d h$ putative null activity alleles, extracted from Australian populations of $D$. melanogaster, all produce some GPDH protein with enzyme activity, and that they are heterogeneous in biochemical properties, unlike the $A d h$ null alleles. The data are compared with those from earlier studies by Lee et al. (1980) and Burkhart et al. (1984) made on Gpdh null alleles isolated by Langley et al. (1981) from natural populations in North America and Europe.

\section{Materials and methods}

Eleven Australian natural populations of $D$. melanogaster, spanning $20^{\circ}$ of latitude, were surveyed between 1983 and 1988 for null and/or low activity alleles at the Gpdh locus (see details in Table 1). The populations were usually sampled between February and August when population sizes were large. All the populations are temporary except at the Coffs Harbour site (a banana/avocado plantation), where a plentiful supply of decaying fruits and mild climate enable $D$. melanogaster to be collected at most times of the year. Elsewhere, the temporary nature of most breeding sites dictates that collections in different years can only be made in the same general locality. In Tasmania it is possible to collect reasonable numbers of $D$. melanogaster during only a few weeks in autumn.

Two methods used to detect variant alleles have been described previously (Freeth \& Gibson, 1985; Gibson et al., 1986) and both methods avoid the effects of $P-M$ hybrid dysgenesis. The method generally used in the present survey involved establishing single female cultures from wild-caught fertilized females and then typing the female and 10 of her progeny for GPDH electrophoretic phenotypes. Putative $G p d h$ null alleles were detected when females, which were typed as homozygotes for one Gpdh allele, produced apparent homozygotes for an alternative $G p d h$ allele amongst their progeny. In other cultures evidence of variant alleles was revealed when abnormal electrophoretic phenotypes occurred amongst the progeny. In some cases these abnormal patterns were produced by heterozygotes for true electrophoretic mobility variants. In other cases the abnormal patterns indicated that the flies carried alleles encoding low GPDH activity and this affected the staining pattern. The criteria used to identify 'null' alleles were essentially the same as those used by Langley et al. (1981) which, as emphasized by Burkhart et al. (1984), are relatively non-stringent. $G p d h$ alleles were isolated from some of the anomalous cultures and made homozygous using $\mathrm{CyO}$ as a second chromosome balancer (and TM3 for the third chromosome), or maintained as hemizygotes with $D f(2 L) G p d h^{A}$ (Lindsley \& Grell, 1969), a deletion which covers $5 \mathrm{cM}$ of the region encompassing the Gpdh locus (Kotarski et al., 1983b).

Electrophoresis of GPDH variants was carried out on single fly homogenates using cellulose acetate membranes and running buffers as previously described (Gibson et al., 1986). In some experiments on isolated variants, thoraces and abdomens were electrophoresed separately. Whole fly homogenates of either of the common electrophoretic variants $(F$ or $S)$ normally produce two main isozymic forms, GPDH-1 and 3 , and an intermediate, weakly staining band, GPDH-2. GPDH-1 is the predominant form in adult flight muscle, whereas GPDH-3 occurs mainly in the abdomen and is the only form present in the larval instars (Niesel et al., 1982).

The levels of GPDH activity in the Gpdh variants isolated from Australian populations were compared with those of the Gpdh null alleles isolated by Langley et al. (1981). Their samples comprised several alleles from a Raleigh, North Carolina, USA population $\langle N C 1, N C 2, N C 3, N C 5, N C 6, N C 7)$ and three alleles isolated from a London, UK population $(G B 1, G B 2$, $G B 3$ ). Stocks of these alleles were obtained from the Mid-America Drosophila Stock Centre at Bowling Green, either as homozygotes or as heterozygotes with $\mathrm{CyO}$.

GPDH activity and GPDH protein amount were measured using the protocols of Bewley et al. (1974) and Lewis \& Gibson (1978) respectively. The antiserum used was prepared in goats to purified GPDH.

\section{Results}

For comparative purposes we followed Langley et al. (1981) and took as evidence for a putative null allele the failure of the wild-derived allele to show normal activity in heterozygotes with one or other of the $G p d h^{F}$ or $G p d h^{S}$ control alleles. On this non-stringent basis the frequencies of Gpdh variants detected in Australian populations ranged from 0.002 in a Tamar population sample to 0.027 in a Cygnet sample (Table 1). Fifty-seven variants were identified from the populations surveyed, giving a frequency of 0.011 . The frequency in the Tasmanian samples (0.013) was not significantly higher $(P>0.05)$ than that in populations on the mainland $(0.009)$. 
The Gpdh variants could be classified into four groups solely on the basis of their electrophoretic phenotypes when heterozygous with the appropriate control alleles, $G p d h^{F}$ and/or $G p d h^{S}$. One group of variants produced novel phenotypes as heterozygotes, indicating that they were alleles that encoded enzyme with altered electrophoretic mobility relative to the control $F$ and $S$ alleles. This was confirmed when homozygotes for the extracted allele or, if lethal, hemizygotes with $D f(2 L) G p d h^{A}$, were electrophoresed and stained for activity. Two different electrophoretic variants of this kind (one of which was detected in two separate single female lines) were found at a frequency of 0.0004 .

A second group of variants (only two were found) appeared to be true null activity variants, as they did not produce any heterodimer enzyme in heterozygotes with either control allele. When single fly homogenates of homozygotes for one of these variants (the other was lethal as a homozygote) were electrophoresed on cellulose acetate membranes, no activity staining was detectable after the normal staining period.

The heterozygote phenotypes of a third category suggested that these variant alleles encoded markedly lower GPDH activity than the control. Homozygotes for these variants gave some activity staining after electrophoresis.
The fourth group, which comprised the most common variants with an overall frequency of 0.114 , were identified because they produced unusual heterozygous phenotypes of a kind which has been previously described at the Gpdh locus (see fig. 1 in Gibson et al., 1986). These variants, when made homozygous, had the same electrophoretic phenotype as one or the other of the control alleles; similar numbers were electrophoretically 'fast' and 'slow' $\left(\chi_{1}^{2}=1.2, P>0.05\right)$.

In order to test this initial classification, 13 of the second chromosomes carrying variant Gpdh alleles were isolated for further study (see Table 2). They included the two alleles with altered electrophoretic mobility, together with samples from the other three categories of variant allele that had been identified. The isolated alleles were assayed for GPDH activity as homozygotes (if viable), as hemizygotes with $D f(2 L)$ $G p d h^{A}$, and as heterozygotes with $G p d h^{F}$ and/or $G p d h^{s}$ control alleles. Sets of crosses producing these genotypes for each variant allele were cultured and assayed concurrently. Within each set, the different genotypes were assayed in random order. In the stocks assayed, the third chromosomes were randomly derived from the wild population so that relative differences in activity are mainly due to second chromosomes bearing the isolated $G p d h$ alleles, although interchromosomal effects on activity cannot be

Table 1 The geographical locations of the Australian populations screened for $G p d h$ variants, and the frequencies of the variant alleles identified

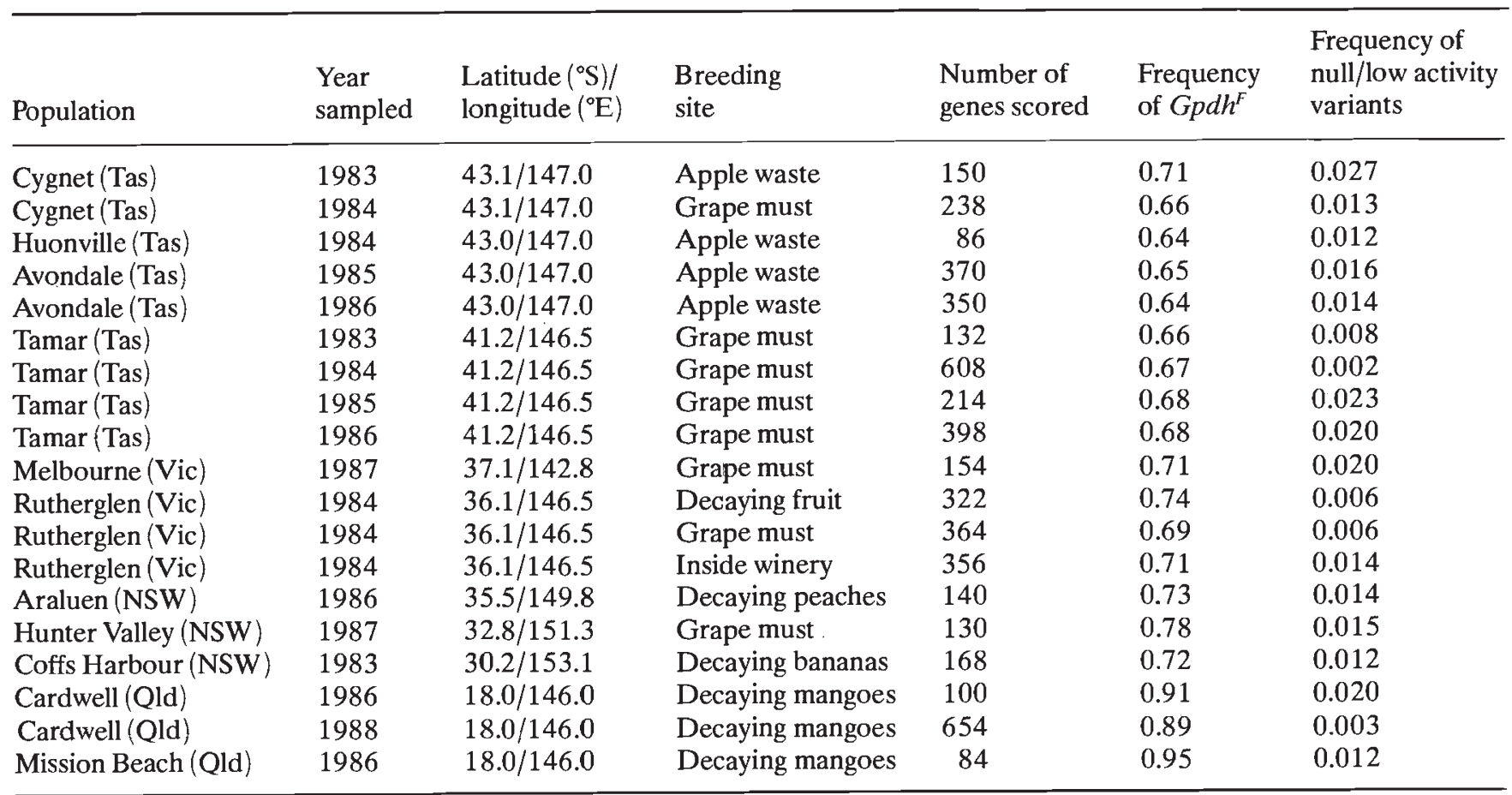


Table 2 GPDH activity ( \pm standard errors) of extracted Gpdh low-activity variants. At least five separate cultures were assayed for each genotype

\begin{tabular}{lllllll}
\hline \multicolumn{5}{l}{ Electrophoretic } & \multicolumn{5}{l}{ Gpdh genotype } \\
\cline { 5 - 7 } Allele & mobility & Population & $+/+$ & $+/ D f(2 L) G p d h^{A}$ & $+/ G p d h^{F}$ & $+/ G p d h^{S}$ \\
\hline Cb62 & $*$ & Cardwell 1986 & Lethal & $40.5 \pm 1.5$ & $276.5 \pm 6.9$ & $281.2 \pm 6.2$ \\
Ky33 & $*$ & Cardwell 1988 & $209.5 \pm 2.7$ & $95.2 \pm 2.9$ & Not assayed & $386.5 \pm 6.4$ \\
Cyg22 & $F$ & Cygnet 1984 & $38.9 \pm 2.5$ & $19.3 \pm 1.7$ & $236.4 \pm 8.6$ & $249.8 \pm 9.4$ \\
MB5 & $F$ & Mission Beach 1986 & $57.1 \pm 2.7$ & $38.9 \pm 1.8$ & $348.9 \pm 2.7$ & $334.6 \pm 11.2 \ddagger$ \\
AV203 & $F$ & Avondale 1986 & Lethal & Lethal & $280.5 \pm 14.0$ & $253.1 \pm 13.8$ \\
Cyg8 & $S$ & Cygnet 1983 & Lethal & $92.1 \pm 2.6$ & $337.0 \pm 9.6$ & $346.1 \pm 8.9$ \\
Cyg83 & $F$ & Cygnet 1984 & Lethal & $91.9 \pm 2.7$ & $370.9 \pm 20.4$ & $379.3 \pm 14.1 \ddagger$ \\
H31 & $F$ & Huonville 1984 & $187.6 \pm 11.7$ & $97.4 \pm 8.4$ & $378.0 \pm 4.4$ & $396.6 \pm 13.2 \ddagger$ \\
AV65 & $S$ & Avondale 1985 & $190.5 \pm 7.9$ & $88.6 \pm 4.3$ & $410.8 \pm 18.9$ & $381.5 \pm 5.3 \ddagger$ \\
AV110 & $S$ & Avondale 1985 & $160.1 \pm 2.7$ & $93.7 \pm 3.6$ & $388.2 \pm 14.0$ & $418.9 \pm 7.7 \ddagger$ \\
AV172 & $S$ & Avondale 1985 & Lethal & $89.9 \pm 3.2$ & $392.7 \pm 1.5$ & $385.5 \pm 4.3 \ddagger$ \\
T198 & $S$ & Tamar 1984 & $179.9 \pm 5.9$ & $87.4 \pm 5.8$ & $382.4 \pm 18.4$ & $384.4 \pm 19.9 \ddagger$ \\
Ko107 & $S$ & Cardwell 1988 & $233.2 \pm 2.3$ & $92.7 \pm 4.7$ & Not assayed & $520.0 \pm 7.1 \ddagger$ \\
Control & $F$ & Cygnet 1984 & $438.5 \pm 10.2$ & $197.0 \pm 7.1$ & & \\
Control & $S$ & Cygnet 1984 & $431.9 \pm 16.9$ & $217.0 \pm 11.6$ & & \\
\hline
\end{tabular}

*See Figs 2 and 3 for electrophoretic phenotypes of $C b 62$ and $K y 33$.

$\dagger$ Assays were made over 2 years later than the others.

$¥$ Activity in heterozygotes significantly higher than expected $(P<0.05)$.

Fig. 1 Electrophoretic phenotypes of $G p d h^{C y g 22}$ and $G p d h^{M B 5}$ compared to the controls. The samples from left are: $G p d h^{F}$ whole adult, thorax, abdomen; Gpdh ${ }^{M B 5}$ whole adult, thorax, abdomen; $G p d h^{C y g 22}$ whole adult, thorax, abdomen; $G p d h^{S}$ whole adult, thorax, abdomen.

discounted (Laurie-Ahlberg \& Bewley, 1983). Five of the 13 alleles were carried on homozygous lethal second chromosomes, and $A V 203$ was lethal as a hemizygote with $D f(2 L) G p d h^{A}$. One of the putative null activity alleles was lethal in heterozygotes with the $\mathrm{CyO}$ chromosome and was not isolated.

A notable feature of these assay data (Table 2) is that all of the $G p d h$ variants isolated produced some GPDH activity and CRM, even Cyg22, which had appeared to be a null allele after electrophoresis and staining (Fig. 1). Cyg22 homozygotes had less than 10 per cent of the activity of the control alleles (Table 2) and the enzyme had the same electrophoretic mobility as GPDH-F (Fig. 1).

Other than for Cyg22, the activity data largely supported the preliminary classification of alleles into the other three categories, based on the electrophoretic phenotypes of heterozygotes. MB5 was confirmed as a low activity variant (about 15 per cent of the normal activity level, Table 2), although GPDH activity in $M B 5 / F$ and $M B 5 / S$ heterozygotes is significantly higher than the mid-parental values, but less than double the control hemizygote (Table 2). AV203 was homozygous lethal, and lethal in heterozygotes with $D f$ (2L) $G p d h^{A}$ but the level of activity in heterozygotes with either $F$ or $S$ suggests that $A V 203$ is also a low activity variant. Cyg8 was also homozygous lethal, but in hemizygotes with $D f(2 L) G p d h^{A}$ it had about 46 per cent of the control allele activity.

The GPDH activity data for the seven extracted alleles of the most common type confirmed that they had low activity (about 47 per cent of normal) and that in heterozygotes between them and either control allele the activity was not significantly lower than twice that of the control hemizygote. The properties of two of these alleles, $T 198$ and $H 31$, have previously been described in more detail by Gibson et al. $(1985,1986)$, who showed that the increase in activity in heterozygotes was apparently due to enhanced expression of the control allele, rather than to increased heterodimer activity.

The two variants with altered electrophoretic mobility have not previously been reported in population surveys of variation at the Gpdh locus. One of 
(a)

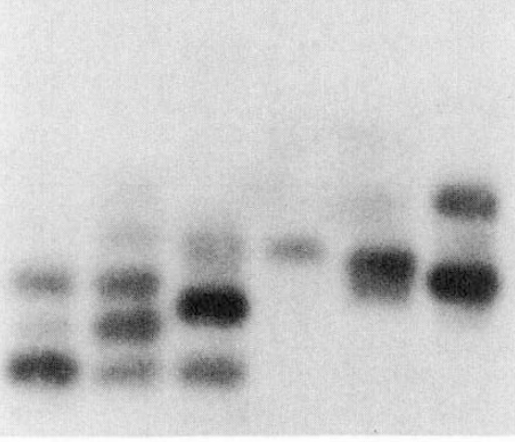

(b)
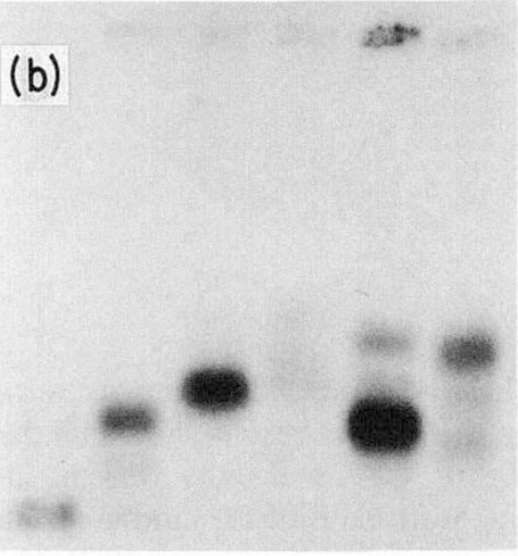

(c)

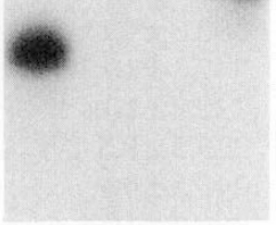

(d)

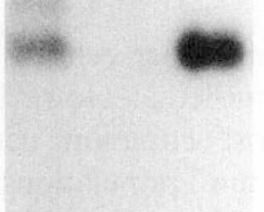

Fig. 2 Electrophoretic phenotypes of $G p d h^{C b 62}$ compared to the controls. A single fly or larva of each genotype was homogenized for each sample except for the hemizygote $G p d h^{C b 62} / D f(2 L) G p d h^{A}$ where two individuals, or parts thereof, were used. (a) Adults (from left) $G p d h^{F} / G p d h^{F}$, $G p d h^{F} / G p d h^{S}, G p d h^{F} / G p d h^{C b 62}, G p d h^{C b 62} / D f(2 L) G p d h^{A}$, $G p d h^{C b 2} / G p d h^{S}, G p d h^{S} / G p d h^{S}$. (b) Adults (from left) $G p d h^{F} /$ $G p d h^{F}$ thorax, abdomen; $G p d h^{C b 6^{2}} / D f(2 L) G p d h^{A}$, thorax, abdomen; $G p d h^{S} / G p d h^{S}$ thorax, abdomen. (c) Third instar larvae (from left) $G p d h^{F} / G p d h^{F}, G p d h^{C b 62} / D f(2 L) G p d h^{A}$ $G p d h^{S} / G p d h^{S}$. (d) Third instar larvae (from left) $G p d h^{F} /$ $G p d h^{C b 62}, G p d h^{C b 62} / D f(2 L) G p d h^{A}, G p d h^{F} / G p d h^{F}$.

these, $C b 62$, had about 20 per cent of the normal level of activity and after electrophoresis of homogenates of whole adult flies the main band of activity stains at a position slightly less anodal than GPDH-S1 (Fig. 2a). There is no indication of the GPDH-2, and very little of the GPDH-3, isozyme normally present in adult flies. Comparison of homogenates of the adult thoraces and adult abdomens shows that, in $\mathrm{Cb62, \textrm {GPDH }}$ is mainly detected in thoracic material and, in contrast to normal alleles, there is little enzyme present in the abdomen (Fig. 2b). In homogenates of Cb62 third instar larvae there is a very weak single band of activity migrating less anodally than GPDH-S3 (Fig. $2 \mathrm{c}$ and d).
Fig. 3 Electrophoretic phenotypes of $G p d h^{K y 33}$ homozygotes compared to the controls. The samples, from left, are: whole adult $G p d h^{S}, G p d h^{F}, G p d h^{K y 33}, G p d h^{K y 33}$, thorax $G p d h^{F}$, abdomen $G p d h^{F}$, (usually more heavily stained) thorax $G p d h^{K y 33}$, abdomen $G p d h^{K y 33}$, third instar larva $G p d h^{F}$, third instar larva $G p d h^{K y 33}$.

The electrophoretic phenotype of $K y 33$ is more complex and difficult to interpret. The major staining activity in adult $K y 33$ homozygotes is at a position analogous to that of GPDH-F3, and the band at the position of GPDH-F1 is very faint. In third instar larvae of Ky33 the single band of activity migrates more anodally than GPDH-F1. However, in thoracic tissue there are two bands of activity, neither of which corresponds in mobility to GPDH-F1 (Fig. 3). The level of GPDH activity in $K y 33$ homozygotes is, in adults, about 40 per cent of that of the controls, but in third instar larvae is 84 per cent.

Overall the 13 extracted variants there was a high correlation $(r=0.91, P<0.01)$ between GPDH activity and immunologically assayed GPDH protein amount.

We have compared GPDH activity levels and other properties of $G p d h$ variants isolated from the Australian populations with those isolated by Langley et al. (1981). Their nine Gpdh null alleles were assayed as hemizygotes with $D f(2 L) G p d h^{A}$ and as heterozygotes with the $G p d h^{S}$ control. These assays were made more than 7 years after their alleles were first isolated, so it is possible that some properties, particularly with respect to GPDH activity levels, have changed. Our interest, however, was not so much the level of activity per se but whether they included variants with similar characteristics to those of the common variant in Australian populations, and this feature is less likely to be altered. The data (Table 3) confirmed the earlier finding of Burkhart et al. (1984) in showing that only one of the nine alleles $(N C 5)$ appears to be a true null activity allele. Two of the alleles, $N C 3$ and $N C 6$, had very low GPDH activity (less than 12 per cent) and the remainder had between 41 and 77 per cent of the activity of the control allele. One allele, GB2, did exhibit the dominance effect found to be characteristic of the most common low-activity variants isolated from Australian populations. 
Table 3 GPDH activity ( \pm standard errors) and CRM in six low-activity alleles extracted from a population in Raleigh, USA and in three alleles from a population in London, UK (see Langley et al., 1981). Five separate cultures of each genotype were assayed

\begin{tabular}{|c|c|c|c|c|}
\hline \multirow{2}{*}{$\begin{array}{l}G p d h \\
\text { allele }\end{array}$} & \multirow{2}{*}{$\begin{array}{l}\text { Electrophoretic } \\
\text { mobility }\end{array}$} & \multirow{2}{*}{$\begin{array}{l}\text { CRM in } \\
\text { homozygote } \\
(\%)\end{array}$} & \multicolumn{2}{|l|}{ GPDH activity } \\
\hline & & & $+\mid D f(2 L) G p d h^{A}$ & $+/ G p d h^{s}$ \\
\hline$N C I$ & UF & 30 & $123.2 \pm 6.7$ & $280.0 \pm 9.1^{*}$ \\
\hline$N C 2$ & $\mathrm{~F}$ & 64. & $177.7 \pm 7.2$ & $347.5 \pm 10.3^{*}$ \\
\hline$N C 3$ & $F$ & 32 & $25.5 \pm 2.1$ & $230.8 \pm 10.4$ \\
\hline$N C 5$ & - & - & Lethal & $223.5 \pm 8.5$ \\
\hline NC6 & $F$ & 22 & $26.3 \pm 3.3$ & $243.5 \pm 9.1$ \\
\hline$N C 7$ & $\mathrm{~F}$ & 42 & $94.0 \pm 4.8$ & $306.0 \pm 10.4$ \\
\hline$G B 1$ & $\mathrm{~S}$ & 47 & $103.8 \pm 5.7$ & $319.7 \pm 11.5$ \\
\hline$G B 2$ & $\mathrm{~F}$ & 41 & $95.4 \pm 5.5$ & $389.2 \pm 8.9 \dagger$ \\
\hline$G B 3$ & $\mathrm{~F}$ & 49 & $136.5 \pm 4.3$ & $319.5 \pm 7.9^{*}$ \\
\hline Control & $\mathrm{S}$ & 100 & $202.7 \pm 3.1$ & $418.3 \pm 12.5$ \\
\hline
\end{tabular}

*Significantly lower than expected $(P<0.05)$.

$\dagger$ Not significantly different from control parent $(P>0.05)$.

\section{Discussion}

These data suggest that null activity alleles at the $G p d h$ locus in D. melanogaster natural populations are rare, as none was detected amongst a total of 5,018 alleles sampled from 11 Australian populations. This result compares with the single $\mathrm{CRM}^{-\mathrm{ve}} G p d h$ allele found by Langley et al. (1981) at a frequency of $8.0 \times 10^{-4}$ in a survey of 1,200 alleles. In a survey of 15,097 $\mathrm{X}$-chromosomes for variants at the glucose-6-phosphate dehydrogenase locus $(G 6 p d)$, Eanes \& Hey (1986) found only one true null activity allele $\left(5.06 \times 10^{-4}\right)$.

All the putative null alleles, which were found in the Australian populations, proved, on further analysis, to be low-activity variants which produced some GPDH CRM with between 10 and 50 per cent of normal levels of activity. The overall frequency of low activity alleles at the $G p d h$ locus ( 1.1 per cent) is similar to that ( 0.83 per cent) reported by Voelker et al. (1980a) and Langley et al. (1981), which was higher than at any of the other 24 allozyme loci investigated in their surveys. This relatively high frequency of $G p d h$ low activity variants in natural populations is surprising in view of evidence linking reduced GPDH activity to viability (O'Brien et al., 1972; Kotarski et al., 1983a). Of the mutants which have less than 30 per cent of the wildtype specific activity, some show lowered flight ability and relative viability, although artificially induced $\mathrm{CRM}^{-\mathrm{ve}} \mathrm{Gpdh}$ mutants are not homozygous lethal (O'Brien \& MacIntyre, 1972). In a study of EMSinduced null mutants, they found that flies with less than 5 per cent of the wild-type GPDH activity were semi-lethal. However, the present results show that the most common category of low-activity variant in natural populations in Australia is of the kind in which heterozygotes with normal activity alleles exhibit dominance in activity, mediated via a trans-acting effect (Gibson et al., 1986). Thus, as homozygotes for these low activity alleles will be rare, the presence of the alleles will have little or no effect on the level of GPDH activity.

It is interesting that a low activity variant with similar properties to the ones most commonly found in Australian populations occurred amongst the variants isolated by Langley et al. (1981) from a natural population in London, UK. This prompts the question whether the properties of these variants arise from the same cause or whether a number of different molecular changes give rise, in populations on different continents, to similar phenotypic effects. The variety of $G p d h$ low-activity variants that we have found was also noted by Burkhart et al. (1984) in their analyses of the material isolated by Langley et al. (1981). They found one variant with altered electrophoretic mobility and we detected two, one of which occurred twice in the same population.

The electrophoretic variant $G p d h^{C b 62}$ is interesting because the enzyme produced is predominantly GPDH-1 and very little is GPDH-3. In homozygotes for normal activity alleles, GPDH-3 comprises approximately one-third of the total activity (Bewley, 1981; Gibson et al., 1986) and is found mainly in the abdomen of adults and in larvae. The low amount of 
GPDH-3 in $G p d h^{C b 62}$ homozygotes might simply be a reflection of the low total enzyme activity rather than deriving from a differential reduction in GPDH-3. The difference in electrophoretic mobility between the enzyme produced by $G p d h^{C b 62}$ and GPDH-S1 is much less than the difference between GPDH-S1 and GPDH-F1. This suggests that the mutation which gave rise to $G p d h^{C b 62}$ alters the structure of the molecule but not its charge state.

Interpretation of the phenotype associated with the second electrophoretic low-activity variant $\left(G p d h^{K y 33}\right)$ is also not straightforward, and its resolution will require biochemical investigations of the adult and larval enzyme forms, together with DNA sequence data, to elucidate their structural relationships.

It seems likely, but as yet unproved, that the mutations responsible for the electrophoretic variants also affect the activity of the enzymes encoded (Collier \& MacIntyre, 1977b). However, in the case of the lowactivity variants which have normal electrophoretic phenotypes, the structural genes may be unaltered and the low activity may result from alterations to linked regulatory sequences. Naturally occurring low GPDH activity variants have previously been described in which the low-activity phenotype segregates as though controlled by a single gene tightly linked to the Gpdh structural gene. Indeed, even in the best studied example, the postulated regulatory element has not been recombined from the Gpdh locus (Shaffer \& Bewley, 1983). Molecular analyses of one of these low activity variants has shown that it produces low levels of a normal-sized mRNA transcript (Cook et al., 1986).

The molecular lesions in the Gpdh low-activity variants isolated in this study can now be elucidated as the Gpdh gene has been cloned and sequenced (Cook et al., 1986, 1988; Bewley et al., 1989; von Kalm et al., 1989). Molecular analyses should help to explain the causes of the heterogeneity of Gpdh low activity variants found at relatively high frequencies in natural populations of $D$. melanogaster. This variety contrasts markedly with the pattern of variation at the $A d h$ locus, in which the naturally occurring null alleles, so far investigated, are probably molecularly homogeneous (Freeth et al., 1987; Gibson \& Wilks, 1989). The differences between the $A d h$ and Gpdh loci in the prevalence and variety of low activity variants is in line with evidence which suggests that the $G p d h$ locus has a higher spontaneous mutation rate (Voelker et al., $1980 \mathrm{~b}$ ), which might be related to differences between the two loci in structure or regulation.

\section{Acknowledgements}

We would like to thank David Sullivan for providing the anti-serum prepared in goats to purified GPDH.

\section{References}

BEWLEY, G. C. 1978 . Heat stability studies at the $\alpha$-glycerophosphate dehydrogenase locus in populations of Drosophila melanogaster. Biochem. Genet., 16, 767-775.

BEWLEY, G. C. 1981. Genetic control of the developmental program of L-glycerol-3-phosphate dehydrogenase isozymes in Drosophila melanogaster. identification of a cisacting temporal element affecting GPDH-3 expression. Dev. Genet., 2, 113-129.

BEWLEY, G. C., COOK, J. L., KUSAKABE, S., MUKAI, T., RIGBY, D. L. AND CHAMBERS, G. K. 1989. Sequence, structure and evolution of the gene coding for $s n$-glycerol-3-phosphate dehydrogenase in Drosophila melanogaster. Nuc. Acids. Res., 17, 8553-8567.

BEWLEY, G. C., RAWLS, J. M., JR. AND LUCCHESI, J. C. 1974. $\alpha-$ glycerophosphate dehydrogenase in Drosophila melanogaster: kinetic differences and developmental differentiation of the larval and adult isozymes. J. Insect. Physiol., 20, 153-165.

BURKHART, B. D., MONTGOMERY, E., LANGLEY, C. H. AND VOELKER, R. A. 1984. Characterization of allozyme null and low activity alleles from two natural populations of Drosophila melanogaster. Genetics, 107, 295-306.

CHAMBERS, G. K., FELTON, A. A., RAMSHAW, J. A. M., RIGBY, D. L. AND SULLIVAN, D. T. 1985. Structural analysis of glycerol-3phosphate dehydrogenase from several Drosophila species. Biochem. Genet., 23, 801-814.

COLLIER, G. E. AND MACINTYRE, R. J. 1977a. Microcomplement fixation studies on the evolution of $\alpha$-glycerophosphate dehydrogenase within the genus Drosophila. Proc. Natl Acad. Sci., USA , 74, 684-688.

COLLIER, G. E. AND MACINTYRE, R. J. 1977b. Low specific activity of rare allozymes of $\alpha$-glycerophosphate dehydrogenase in Drosophila. Nature, 267, 839-841.

COOKE, J. L., BEWLEY, G. C. AND SHAFFER, J. B. 1988. Drosophila $s n$-glycerol-3-phosphate dehydrogenase isozymes are generated by alternate pathways of RNA processing resulting in different carboxy L-terminal amino acid sequences. J. Biol. Chem., 263, 10858-10864.

COOK, J. L., SHAFFER, J. B., BEWLEY, G. C., MACINTYRE, R. J. AND WRIGHT, D. A. 1986. Isolation of a genomic clone for Drosophila sn-glycerol-3-phosphate dehydrogenase using synthetic oligonucleotides. J. Biol. Chem., 261, 11751-11755.

COYNE, J. A., EANES, W. F., RAMSHAW, J. A. M. AND KOEHN, R. K. 1979. Electrophoretic heterogeneity of $\alpha$-glycerophosphate dehydrogenase among many species of Drosophila. Syst. Zool., 28, 164-175.

EANES, W. F. AND HEY, J. 1986. In vivo function of rare G6pd variants from natural populations of Drosophila melanogaster. Genetics, 113, 679-693.

FREETH, A. L. AND GIBSON, J. B. 1985. Alcohol dehydrogenase and $s n$-glycerol-3-phosphate dehydrogenase null activity alleles in natural populations of Drosophila melanogaster. Heredity, 55, 369-374.

FREETH, A. L., GIBSON, J. B. AND DE COUET, H. G. 1986. The partial characterization of alcohol dehydrogenase null alleles from natural populations of Drosophila melanogaster. 
Biochem. Genet., 24, 957-972.

FREETH, A. L., GIBSON, J. B. AND WILKS, A. v. 1987. Transcription analysis of alcohol dehydrogenase null alleles from natural populations of Drosophila melanogaster. Genome, 30, 25-30.

GIBSON, J. B. AND WILKS, A. v. 1989. Molecular structure of a naturally occurring alcohol dehyrogenase null activity allele in Drosophila melanogaster. Biochem. Genet., 27, 679-688.

GIBSON, J. B., WILKS, A. V., CAO, A. AND FREETH, A. L. 1985. Dominance for enzyme activity in Drosophila melanogaster. Experientia, 42, 191-192.

GIBSON, J. B., WILKS, A. V., CAO, A. AND FREETH, A. L. 1986. Dominance for $s n$-glycerol-3-phosphate dehydrogenase activity in Drosophila melanogaster: evidence for differential allelic expression mediated via a trans-acting effect. Heredity, 56, 227-235.

GRELL, E. H. 1967. Electrophoretic variants of $\alpha$-glycerophosphate dehydrogenase in Drosophila melanogaster. Science, 158, 1319-1320.

JIANG, C., GIBSON, J. B. AND CHEN, J. 1989. Genetic differentiation in populations of Drosophila melanogaster from the Peoples' Republic of China: comparison with patterns on other continents. Heredity, 62, 193-198.

JIANG, C., GIBSON, J. B., WILKS, A. V. AND FREETH, A. L. 1988. Restriction endonuclease variation in the region of the alcohol dehydrogenase gene: a comparison of null and normal alleles from natural populations of Drosophila melanogaster. Heredity, 60, 101-107.

JOHNSON, F. M. AND SCHAFFER, J. E. 1973. Isozyme variability in species of the genus Drosophila. VII. Genotype-environment relationships in populations of D. melanogaster from the eastern United States. Biochem. Genet., 10, 149-163.

KOTARSKI, M. A., PICKERT, S., LEONARD, D. A., LA ROSA, G. T. AND MACINTYRE, R. J. 1983a. The characterization of $\alpha$-glycerophosphate dehydrogenase mutants in Drosophila melanogaster. Genetics, 105, 387-407.

KoTARSKI, M. A., PICKERT, S. AND MACINTYRE, R. J. 1983b. A cytogenetic analysis of the chromosomal region surrounding the $\alpha$-glycerophosphate dehydrogenase locus of Drosophila melanogaster. Genetics, 105, 371-386.

LANGLEY, C. H., VOELKER, R. A., LEIGH BROWN, A. J., OHNISH, S., DiCKSON, B. AND MONTGOMERY, E. 1981. Null allele frequencies at allozyme loci in natural populations of Drosophila melanogaster. Genetics, 99, 151-156.

LAURIE-AHLBERG, C. C. AND BEWLEY, G. C. 1983. Naturally occurring genetic variation affecting the expression of $s n^{-}$ glycerol-3-phosphate dehydrogenase in Drosophila melanogaster. Biochem. Genet., 21, 943-961.

LEE, C.-Y., NIESEL, D. AND BEWLEY, G. C. 1980. Analyses of genetic variants of $\alpha$-glycerol-3-phosphate dehydrogenase in Drosophila melanogaster by two-dimensional gel electrophoresis and immunoelectrophoresis. Biochem. Genet., 18, 1003-1018.

LEWIS, N. AND GIBSON, J. 1978. Variation in amount of enzyme protein in natural populations. Biochem. Genet., 16, $159-170$.

LINDSLEY, D. L. AND GRELL, E. H. 1968. Genetic variations of Drosophila melanogaster. Carn. Inst. Wash. Pub., Number 627.

NIESEL, D. W., PAN, Y.-C. E.,, BEWLEY, G. C., ARMSTRONG, F. B. AND LI, S. S.-L. 1982. Structural analysis of adult and larval isozymes of $s n$-glycerol-3-phosphate dehydrogenase of Drosophila melanogaster. J. Biol. Chem., 257, 979-983.

OAKESHOTT, J. B., GIBSON, J. B., ANDERSON, P. R., KNIBB, W. R., ANDERSON, D. G. AND CHAMBERS, G. K. 1982. Alcohol dehydrogenase and glycerol-3-phosphate dehydrogenase clines in Drosophila melanogaster on different continents. Evolution, 36, 86-96.

O'BRIEN, S. J. AND MACINTYRE, R. J. 1972. The $\alpha$-glycerophosphate cycle in Drosophila melanogaster. II. Genetic aspects. Genetics, 71, 127-138.

o'BriEn, S. J., WAllace, B. AND MACintyre, R. J. 1972. The $\alpha$ glycerophosphate cycle in Drosophila melanogaster. III. Relative viability of 'null' mutants at the $\alpha$-glycerophosphate dehydrogenase-1 locus. Am. Nat, 106, 767-771.

otto, J., ARgos, P. AND Rossmann, M. G. 1980. Prediction of secondary structural elements in glycerol-3-phosphate dehydrogenase by comparison with other dehydrogenases. Eur. J. Biochem. 109, 325-330.

SACKTOR, B. 1970. Regulation of intermediary metabolism with special reference to control mechanisms in insect flight muscle. Adv. Insect. Physiol. 7, 267-347.

SHAFFER, J. B. AND BEWLEY, G. C. 1983. Genetic determination of $s n$-glycerol-3-phosphate dehydrogenase synthesis in Drosophila melanogaster. J. Biol. Chem., 258 , 10027-10033.

SINGH, R. S., HICKEY, D. A. AND DAVID, J. 1982. Genetic differentiation between geographically distant populations of Drosophila melanogaster. Genetics, 101, 235-256.

VOELKER, R. A., LANGLEY, C. H., LEIGH BROWN, A. J., OHNISHI, S., DICKSON, B., MONTGOMERY, E. AND SMITH, s. 1980a. Enzyme null alleles in natural populations of Drosophila melanogaster: frequencies in a North Carolina population. Proc. Natl Acad. Sci. USA, 77, 1091-1095.

VOELKER, R. A., SCHAFFER, H. E. AND MUKAI, T. 1980b. Spontaneous allozyme mutations in Drosophila melanogaster: rate of occurrence and nature of the mutants. Genetics, 94, 961-968.

VON KALM, L., WEAVER, J., DEMARCo, J., MACINTYRE, R. J. AND SULLIVAN, D. T. 1989 . Structural characterization of the $\alpha$ glycerol-3-phosphate dehydrogenase-encoding gene of Drosophila melanogaster. Proc. Natl Acad. Sci. USA, 86, 5020-5024. 